\title{
Noninvasive brain stimulation in combination with psychotherapy for anxiety disorders.
}

\section{A systematic review}

\section{Herrmann, M.J., Cybinski, L.M. Unterecker, S., Deckert, J., Polak, T.}

Center of Mental Health, Department of Psychiatry, Psychosomatics, and Psychotherapy, University Hospital of Würzburg, Würzburg, Germany

\section{Correspondence}

Prof Dr M. J. Herrmann, Department of Psychiatry, Psychosomatics, and Psychotherapy, University Hospital of Würzburg, Margarete-Hoeppel-Platz 1, D-97080 Würzburg, Germany. Email: herrmann_m@ukw.de

\section{Summary}

Background: Although anxiety disorders can be treated very well with cognitive behavioral therapy, there are some patients who are not yet optimally benefiting from the therapy. In recent years, noninvasive brain stimulation methods have been studied as an add-on to psychotherapy.

Objectives. Is there sufficient evidence for the use of noninvasive brain stimulation in the psychotherapy of anxiety disorders?

Methods. A systematic review of previous therapy studies reviewing noninvasive brain stimulation in cognitive behavioral therapy of anxiety disorders.

Results. In total, only 4 randomized controlled therapeutic trials could be found and were analyzed. These studies used repetitive transcranial magnetic stimulation (rTMS) shortly before or during exposure-based behavioral therapy. The use of active stimulation compared to placebo stimulation shows a positive additional effect in the medium range.

Conclusions. Initial work shows an additional benefit of rTMS in cognitive behavioral therapy for the treatment of anxiety disorders. However, further studies must show which exact stimulation parameters are best suited to make a recommendation for the treatment practice

Keywords. Noninvasive brain stimulation, tDCS, rTMS, anxiety disorders, PTSD, phobia 
Anxiety disorders represent a relevant group of mental illnesses that can be treated very well with cognitive behavioral therapy. In order to further optimize exposure therapy, non-invasive brain stimulation methods are currently being investigated in scientific studies to support specific psychological processes in the therapeutic approach. In this systematic review, we summarize all previous studies that investigate brain stimulation in conjunction with psychotherapy for anxiety disorders.

\section{Introduction}

\section{Anxiety Disorders}

More than $15 \%$ of the population suffer from an anxiety disorder at least once in their lives (Jacobi et al., 2014). This means that the frequency of anxiety disorders is even higher than that of affective disorders and disorders caused by substance abuse. Considering the global burden of disease, expressed as "disabilityadjusted life years" (dalys), patients with anxiety disorders show the second highest burden of mental illness after patients with depressive disorders (Whiteford et al., 2013).

Although cognitive behavioral therapy (CBT) is already very effective for anxiety disorders (Carpenter et al., 2018), not all patients benefit sufficiently from such treatment. For example, in the area of panic disorder, about $40 \%$ of patients did not show an adequate response to therapy (Gloster et al., 2011). Further development of therapy methods is necessary and is being pursued in both large-scale multi-centre therapy studies (Arolt et al., 2009; Gloster et al., 2011) and also in experimental approaches (Shiban et al., 2017; Shiban et al., 2015). In addition, non-invasive brain stimulation procedures (NIBS) are being increasingly investigated as a complement to psychotherapy and psychopharmacological therapy (Bajbouj and Padberg, 2014; Zwanzger et al., 2016). The theoretical basis for this is provided, among other things, by imaging studies that have demonstrated changes in neuronal activation through psychotherapy (Brooks and Stein, 2015; Kircher et al., 2013; Lueken et al., 2013). From this, the question can be deduced whether additional modulation of these brain areas by suitable non-invasive brain stimulation procedures can achieve a positive therapeutic effect.

\section{Non-invasive brain stimulation}

Non-invasive brain stimulation procedures include transcranial magnetic stimulation (TMS) and transcranial direct current stimulation (tDCS). In TMS, a magnetic impulse is applied to the surface of the skull, which leads to an electrical current flow in the brain tissue and subsequently triggers depolarization of the neurons and action potential. The repetitive TMS (rTMS) is a repeated stimulation of cortical areas. This results in an inhibitory influence at slow $(1 \mathrm{~Hz})$ and an excitatory influence at fast (from $5 \mathrm{~Hz}$ ) stimulation frequency of the respective area (Lang and Siebner, 2007). In tDCS, a weak direct current is applied which flows between the anodal and cathodal electrodes. No action potentials are triggered, but a shift of the resting membrane potentials is caused. The anodal stimulation leads to a facilitated activation, the cathodic stimulation to a reduced activation (Nitsche and Paulus, 2000).

Two systematic reviews about the treatment of patients with panic disorder (Li et al., 2014) and posttraumatic stress disorder (Berlim and Van Den Eynde, 2014) by brain stimulation procedures show a positive effect of rTMS. However, the question of optimal stimulation localization, whether activating or inhibitory brain stimulation is more advantageous and, ultimately, whether and how a combination with psychotherapy makes sense remains open. Marin and colleagues (Marin et al., 2014) suggest that future studies should increasingly focus on therapy-relevant processes, such as fear extinction, which is an essential process in the exposure therapy of anxiety disorders (Vervliet et al., 2013). Initial studies have shown that both rTMS (Guhn et al., 2014; Raij et al., 2018) and tDCS (Dittert et al., 2018) can modulate fear extinction.

This systematic review integrates previous studies on the direct combination of brain stimulation procedures with psychotherapy for anxiety disorders. 


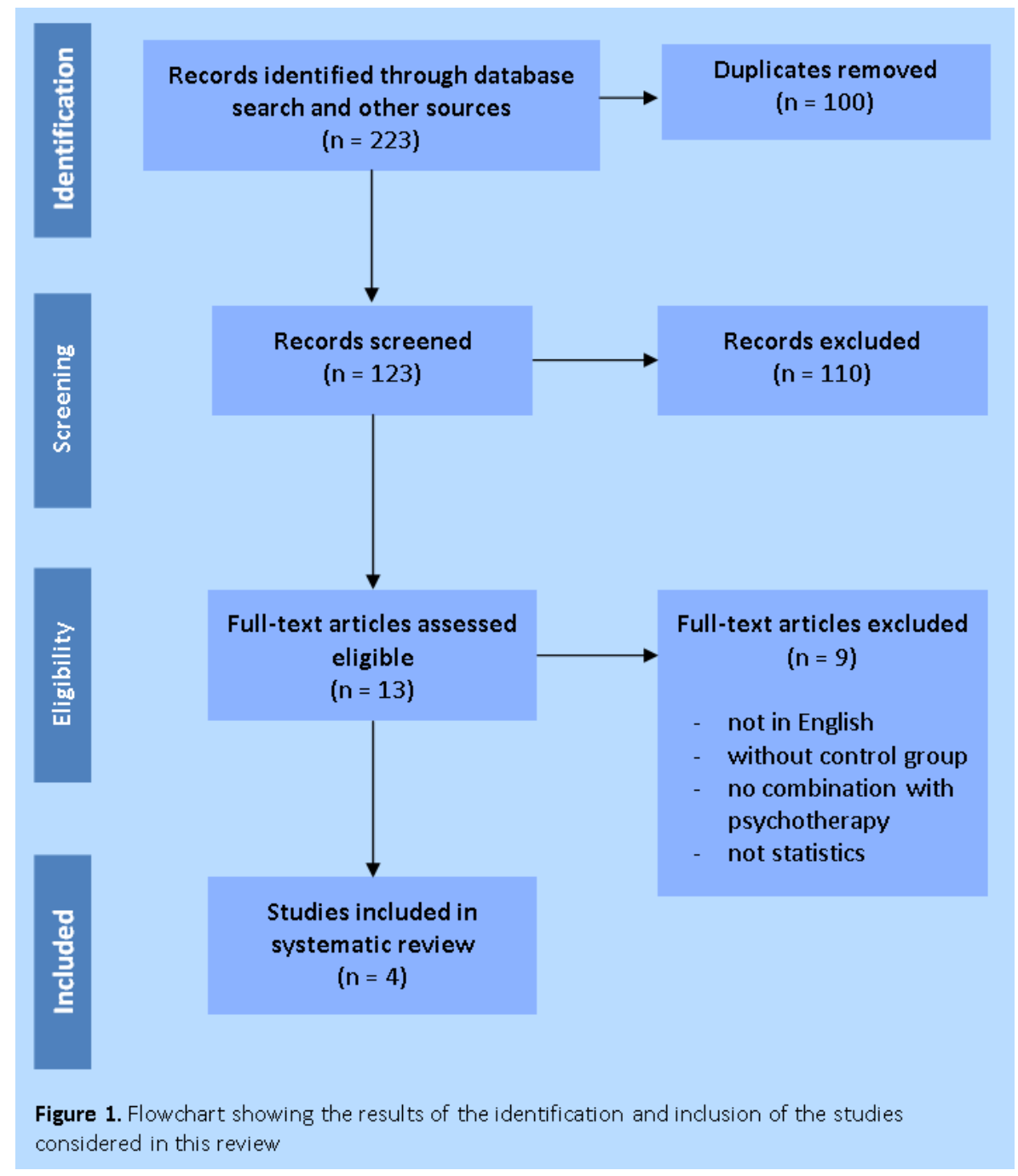

\section{Review}

\section{Search strategy}

The systematic literature search included the databases Pubmed, CENTRAL, Web of Science, Ovid MEDLINE and PsychINFO and was carried out in October 2018 with the following search terms: (psychotherapy or "exposure therapy" or "add on" or augmentation or extinction) AND ("anxiety disorder" OR phobia OR GAS OR panic OR PTSD) AND (tdcs OR tms OR rtms OR NIBS). 223 references were identified, after removing duplicates 123 publications remained available for pre-selection (see flow chart in Figure 1). The pre-selection was carried out in parallel by two independent persons using the following inclusion and exclusion criteria:

\section{Inclusion criteria:}

- written in English or German language

- patients with diagnosed anxiety disorder were examined (ICD-10: F40, F41)

- psychotherapy was performed

- the study was carried out on humans

- only adult patients were enrolled

\section{Exclusion criteria}

- review, conference papers, commentary

13 papers were then tested for suitability in full text. Nine of the papers had to be excluded so that a total of four therapy studies could be included. One paper was written in Spanish, one paper did not include a control group, five papers did not include brain stimulation in a close temporal relationship to psychotherapy, and two other papers were to be evaluated only as feasibility studies, as these could only include up to five patients per therapy arm.

\section{Results}

Table 1 lists the studies included in this review. All papers use transcranial magnetic stimulation as a noninvasive brain stimulation method, with three papers using rTMS and one paper using a further development called deep TMS. In deep TMS, the coils for generating the magnetic field are attached in a complex form so that the stimulation effects can reach deeper brain areas as well (Zangen et al., 2005). Three of the studies examined patients with post-traumatic stress disorder (PTSD), one included patients with a specific phobia. Both for PTSD (Mühlberger and Voderholzer, 2015) and for a specific phobia (Bandelow et al., 2014) exposure-based therapy approaches are the procedure of choice, which in turn are partly based on extinction learning (Vervliet et al., 2013). 


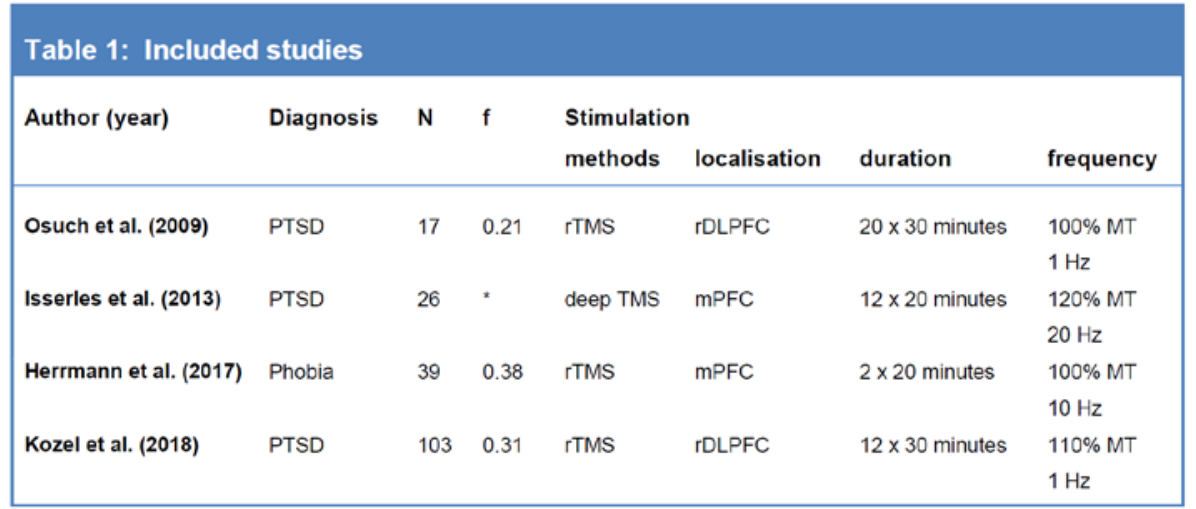

$\mathrm{N}=$ number of patients included; $\mathrm{f}=$ effect strength $\mathrm{f}$ for the interaction treatment group (verum, placebo) $\times$ time (prepost); " not calculable due to data in the publication.

PTSD = post-traumatic stress disorder, rTMS = repetitive transcranial magnetic stimulation; rDLPFC= right dorsolateral prefrontal cortex; $\mathrm{mPFC}=$ medial prefrontal cortex; $\mathrm{MT}=$ motor threshold; $\mathrm{Hz}=$ frequency in Hertz

It is therefore obvious that the work of Isserles et al. (2013) and Herrmann et al. (2017) both define the medial prefrontal cortex (mPFC) as a target region since it plays an important role in extinction learning (Fullana et al., 2018). Both studies use repetitive TMS to activate the mPFC with the aim to augment the extinction learning during exposure therapy. In the first study (Isserles et al., 2013), a total of 26 patients with therapy-resistant PTSD were randomized to three therapy groups. One group received only rTMS $(\mathrm{N}=8)$, one group received placebo stimulation ( $\mathrm{N}=9$ ) and one group received rTMS in addition to the exposure sessions $(N=9)$. The exposure was based on an individualized audio script of approximately 30 seconds length. Exposure was performed in sensu for 20 minutes. A total of 12 sessions were completed in 4 weeks. The effect size in the group with exposure sessions and rTMS was $f=0.58$, in the rTMS only group $f=0.18$ and in the exposure session only group with placebo stimulation $f=0.16$. The study was performed as a randomized double-blind therapy study. Only the group combined with TMS, the effect size was $\mathrm{f}=0.57$ for the "Anxiety" scale and $f=0.58$ for the "Avoidance" scale. For the interaction group $x$ time, an effect size of $f=0.38$ was found for the scale "Anxiety" and "Avoidance", respectively. The drop-out rate was only $7 \%$ and was distributed equally across both treatment groups. The side effects reported in the active stimulation group were headache in 7 patients and drowsiness in one person. In the placebo-stimulated group, two patients reported neck pain and one person headache.

The other two studies (Kozel et al., 2018; Osuch et al., 2009) apply an inhibitory rTMS over the right DLPFC. Although the theoretical justification for this approach is not explicitly mentioned in the papers, the studies are based on preliminary results that have found a reduction of amygdala activity to threatening stimuli after $1 \mathrm{~Hz}$ rTMS of rDLPFC (Baeken et al., 2010). In the work of Kozel et al. (2018) with a total of 103 PTSD patients, an effect size of $f=0.31$ was found for the significant interaction group $x$ time (time 1 month after end of treatment) for the external assessment instrument CAPS (Blake et al., 1995). Here again, the decrease in symptoms was significantly stronger in the verumstimulated group than in the placebostimulated group. The psychotherapeutic treatment included exposure sessions as well as cognitive elements and was performed in both groups once a week over a period of 12 weeks. TMS was performed for 30 minutes, followed by a 
psychotherapeutic session for 60 minutes.

This study was a randomized doubleblind therapy study as well in which only the user of the TMS knew the assignment to the stimulation conditions. The drop-out rate was 32\% and was equally distributed across both groups. Headaches were reported as side effects in the active stimulation group of two patients and in the placebo stimulation group of one patient.

The next study, which combined psychotherapy with brain stimulation in the area of anxiety disorders, was published by Osuch et al. (2009). In this study, patients with chronic PTSD were treated with individualized gradual exposure therapy over 15 sessions. At the same time, patients received either an active or a placebo $1 \mathrm{~Hz}$ rTMS over the rDLPFC for 30 minutes. In total, only 9 patients in a crossover design were enrolled in the double-blind randomized study. One patient discontinued the study prematurely. No side effects were reported. The decrease of the hyperarousal subscale of CAPS was greater in the active TMS group than in the placebo-stimulated group with an effect size of $f=0.21$. The reduction of the hyperarousal subscale of CAPS was greater in the active TMS group than in the placebo-stimulated group.

\section{Discussion}

In summary, it can be concluded that non-invasive brain stimulation can be well combined with psychotherapeutic procedures in the treatment of anxiety disorders. The mean, weighted effect strength of such a procedure in comparison to a purely psychotherapeutic procedure with placebo stimulation lies with $f=0.32$ in the range of a medium ( $f=0.25$ ) to large $(f=0.40)$ effect (Ellis, 2010). The discontinuation rate differs greatly between the studies and ranges from $7 \%$ to $32 \%$. There are also clear differences between the studies in the area of side effects. In studies with rTMS applications, there is no difference between the verum- and placebo-stimulated groups. The side effects are in the range of mild headaches. The epileptic seizure that occurred with deep TMS can be considered a serious side effect. To what extent the activating stimulation of the medial PFC or the inhibitory stimulation of the rDLPFC will prove to be more effective will have to be investigated in future studies. The question of the extent to which medial PFC can be achieved with TMS cannot be answered in this review. Yet it is certain that deep TMS can reach deeper areas of the brain than standard TMS. It has also been shown that stimulation is not limited to the directly stimulated cortical area, but can reach deeper areas, such as the medial PFC, via functional coupling (Raij et al., 2018). Recent publications show that the tDCS method also improves extinction in healthy controls (Dittert et al., 2018) and can be combined with exposure procedures in PTSD (van 't Wout-Frank et al., 2019). Therefore, new randomized and controlled therapy studies will certainly be published with this method in the future. Furthermore, it is obvious to augment exposure therapies for other anxiety disorders with non-invasive brain stimulation.

Conclusion for the practice

- Brain stimulation procedures for the augmentation of cognitive behavioral therapy in anxiety disorders are currently still in an experimental stage.

- Both basic studies on the modulation of fear extinction and first randomized controlled therapy studies show an additional benefit (mean weighted effect strength $f=$ 0.32) of brain stimulation for exposure-based therapy in anxiety disorders.

- These initial positive results are encouraging, but additional randomized trials in various anxiety disorders must show which precise stimulation parameters are best suited to make a recommendation for treatment practice.

Conflict of interest. The authors state that there is no conflict of interest.

This article does not include primary data from studies on humans or animals.

\section{References}

Arolt, V, Zwanzger, P, Strohle, A et al. (2009) [The research network PANIC-NET: improving the treatment of panic disorder - from a better understanding of fear circuit mechanisms to more effective psychological treatment and routine care]. Psychotherapie, Psychosomatik, medizinische Psychologie, 59: 124-131

Baeken, C, De Raedt, R, Van Schuerbeek, P et al. (2010) Right 
prefrontal HF-rTMS attenuates right amygdala processing of negatively valenced emotional stimuli in healthy females. Behavioural brain research, 214: 450-455

Bajbouj, M, Padberg, F (2014) A perfect match: noninvasive brain stimulation and psychotherapy. European archives of psychiatry and clinical neuroscience, 264 Suppl 1: S27-33

Berlim, MT, Van Den Eynde, F (2014) Repetitive transcranial magnetic stimulation over the dorsolateral prefrontal cortex for treating posttraumatic stress disorder: an exploratory meta-analysis of randomized, double-blind and sham-controlled trials. Canadian journal of psychiatry. Revue canadienne de psychiatrie, 59: 487-496

Blake, DD, Weathers, FW, Nagy, LM et al. (1995) The development of a Clinician-Administered PTSD Scale. Journal of traumatic stress, 8: $75-90$

Brooks, SJ, Stein, DJ (2015) A systematic review of the neural bases of psychotherapy for anxiety and related disorders. Dialogues in clinical neuroscience, 17: 261-279

Carpenter, JK, Andrews, LA, Witcraft, SM et al. (2018) Cognitive behavioral therapy for anxiety and related disorders: A meta-analysis of randomized placebo-controlled trials. Depression and anxiety, 35: 502-514

Cohen, DC (1977) Comparison of selfreport and overt-behavioral procedures for assessing acrophobia. Behavior therapy, 8: 17-23

Dittert, N, Huttner, S, Polak, T et al. (2018) Augmentation of Fear Extinction by Transcranial Direct Current Stimulation (tDCS). Frontiers in behavioral neuroscience, 12: 76

Ellis, PD, 2010. The essential guide to effect sizes: Statistical power, meta-analysis, and the interpretation of research results. Cambridge University Press, Cambridge, New York.

Fullana, MA, Albajes-Eizagirre, A, Soriano-Mas, C et al. (2018) Fear extinction in the human brain: $A$ meta-analysis of fMRI studies in healthy participants. Neuroscience and biobehavioral reviews, 88: 1625
Gloster, AT, Wittchen, HU, Einsle, F et al. (2011) Psychological treatment for panic disorder with agoraphobia: a randomized controlled trial to examine the role of therapist-guided exposure in situ in CBT. Journal of consulting and clinical psychology, 79: 406-420

Guhn, A, Dresler, T, Andreatta, $M$ et al. (2014) Medial prefrontal cortex stimulation modulates the processing of conditioned fear. Frontiers in behavioral neuroscience, 8: 11

Herrmann, M.J., Cybinski, L.M. Unterecker, S., Deckert, J., Polak, T. (in press) Nichtinvasive Hirnstimulation in Kombination mit Psychotherapie bei Angsterkrankungen. Systematisches Review. Psychotherapeut; doi.org/10.1007/s00278-019-0349$x$

Herrmann, MJ, Katzorke, A, Busch, Y et al. (2017) Medial prefrontal cortex stimulation accelerates therapy response of exposure therapy in acrophobia. Brain stimulation, 10: 291-297

Isserles, M, Shalev, AY, Roth, Y et al. (2013) Effectiveness of Deep Transcranial Magnetic Stimulation Combined with a Brief Exposure Procedure in Post-Traumatic Stress Disorder - A Pilot Study. Brain stimulation, 6: 377-383

Jacobi, F, Hofler, M, Strehle, J et al. (2014) [Mental disorders in the general population : Study on the health of adults in Germany and the additional module mental health (DEGS1-MH)]. Der Nervenarzt, 85: 77-87

Kircher, T, Arolt, V, Jansen, A et al. (2013) Effect of cognitivebehavioral therapy on neural correlates of fear conditioning in panic disorder. Biol Psychiatry, 73: 93-101

Kozel, FA (2018) Clinical Repetitive Transcranial Magnetic Stimulation for Posttraumatic Stress Disorder, Generalized Anxiety Disorder, and Bipolar Disorder. The Psychiatric clinics of North America, 41: 433446

Kozel, FA, Motes, MA, Didehbani, $\mathrm{N}$ et al. (2018) Repetitive TMS to augment cognitive processing therapy in combat veterans of recent conflicts with PTSD: a randomized clinical trial. Journal of affective disorders, 229: 506-514
Lang, N, Siebner, HR, 2007. Repetitive transkranielle Magnet stimulation, in: Siebner, H., Ziemann, U. (Eds.), Das TMS-Buch. Handbuch der transkraniellen Magnetstimulation. Springer, Heidelberg, pp. 500-511.

Li, H, Wang, JJ, Li, CB et al. (2014) Repetitive transcranial magnetic stimulation (rTMS) for panic disorder in adults. Cochrane Database of Systematic Reviews,

Lueken, U, Straube, B, Konrad, C et al. (2013) Neural substrates of treatment response to cognitivebehavioral therapy in panic disorder with agoraphobia. The American journal of psychiatry, 170: 1345-1355

Marin, M-F, Camprodon, JA, Dougherty, DD et al. (2014) Device-based brain stimulation to augment fear extinction: implications for PTSD treatment and beyond. Depression and anxiety, 31: 269-278

Mühlberger, M, Voderholzer, U (2015) Konfrontationstherapie im Fokus. Wirkungsweise

Besonderheiten

Expositionsverfahren. InFo Neurologie \& Psychiatrie, 17: 36-46

Nitsche, MA, Paulus, W (2000) Excitability changes induced in the human motor cortex by weak transcranial direct current stimulation. The Journal of physiology, 527 Pt 3: 633-639

Osuch, EA, Benson, BE, Luckenbaugh, DA et al. (2009) Repetitive TMS combined with exposure therapy for PTSD: a preliminary study. Journal of anxiety disorders, 23: 54-59

Raij, T, Nummenmaa, A, Marin, MF et al. (2018) Prefrontal Cortex Stimulation Enhances Fear Extinction Memory in Humans. Biol Psychiatry, 84: 129-137

Shiban, Y, Diemer, J, Muller, J et al. (2017) Diaphragmatic breathing during virtual reality exposure therapy for aviophobia: functional coping strategy or avoidance behavior? a pilot study. BMC psychiatry, 17: 29

Shiban, Y, Schelhorn, I, Pauli, P et al. (2015) Effect of combined multiple contexts and multiple stimuli exposure in spider phobia: A randomized clinical trial in virtual reality. Behav Res Ther, 71: 45-53

van 't Wout-Frank, M, Shea, MT, Larson, VC et al. (2019) Combined transcranial direct current 
stimulation with virtual reality exposure for posttraumatic stress disorder: Feasibility and pilot results. Brain stimulation, 12: 41-43

Vervliet, B, Craske, MG, Hermans, D (2013) Fear extinction and relapse: state of the art. Annual review of clinical psychology, 9: 215-248

Whiteford, HA, Degenhardt, L, Rehm, $\mathrm{J}$ et al. (2013) Global burden of disease attributable to mental and substance use disorders: findings from the Global Burden of Disease Study 2010. Lancet, 382: 15751586

Zangen, A, Roth, Y, Voller, B et al. (2005) Transcranial magnetic stimulation of deep brain regions: evidence for efficacy of the H-coil. Clin Neurophysiol, 116: 775-779

Zwanzger, P, Herrmann, MJ, Baeken, C (2016) "Torpedo" for the brain: perspectives in neurostimulation. $\mathrm{J}$ Neural Transm (Vienna), 123:

1119-1120 